\title{
Measuring the fluctuation-dissipation ratio in glassy systems with no perturbing field
}

\author{
F. Ricci-Tersenghi \\ Dipartimento di Fisica, SMC and INFM, Università di Roma "La Sapienza", P.le Aldo Moro 2, 00185 Roma, Italy
}

(Dated: November 22, 2018)

\begin{abstract}
A method is presented for measuring the integrated response in Ising spin system without applying any perturbing field. Large-scale simulations are performed in order to show how the method works. Very precise measurements of the fluctuation-dissipation ratio are presented for 3 different Ising models: the 2-dimensional ferromagnetic model, the mean-field diluted 3-spin model, and the 3-dimensional Edwards-Anderson model.
\end{abstract}

Disordered and frustrated models are a fascinating but still poorly understood subject in contemporary statistical mechanics. The interest in these systems also comes from their many interdisciplinary applications: from the physics of glass-former liquids to that of polymers and biomolecules, from the description of error correcting codes to the study of the computational complexity and phase transitions in theoretical computer science.

Here we will use the term glassy system for a generic model showing very slow relaxation to equilibrium [1]. Because of the huge equilibration time, a glassy system may be in the out of equilibrium regime for all the experimental times. Then a complete understanding of this regime is what one needs in order to correctly describe a real slow-evolving material. Moreover, numerical studies of the off-equilibrium regime do not suffer from finite-size effect since very large sizes can be used. They present finite time corrections which can be usually kept under control, thus allowing for better numerical estimations.

Among the numerical methods that can be used in the out of equilibrium regime, the study of the so-called fluctuation-dissipation ratio (FDR) 2] has been shown to be a very successful one $[3,4]$. This method is based on the comparison of how spontaneous and induced fluctuations relax. Actually one measures an autocorrelation function $C(t, s)$ [12] and the associated response function $R(t, s)$ and defines the FDR $X(t, s)$ through the formula

$$
T R(t, s)=X(t, s) \partial_{s} C(t, s)
$$

where $T$ is the temperature. At equilibrium the fluctuation dissipation theorem (FDT) holds, implying $X=1$.

In the large times limit $-s, t \rightarrow \infty$ with $C(t, s) \rightarrow q$ - the FDR $X(t, s)$ converges to the limiting function $X(q)$. The physical meaning of the function $X(q)$ has been explained in Refs. [5], where it has been shown that under some hypothesis (stochastic stability) the equation

$$
X(q)=x(q) \equiv \int_{0}^{q} P\left(q^{\prime}\right) d q^{\prime}
$$

holds. In Eq.(2) $P(q)$ represents the overlap pdf in the threshold states, that is the states reached by the out of equilibrium dynamics on very large times, which could be different from the thermodynamical state 1]. It has been conjectures that the effective temperature $T_{\text {eff }}=T / X$ plays a central role in off-equilibrium glassy systems [6].

In numerical simulations the punctual response function $R(t, s)$ is very noisy, while a much better signal can be obtained for the integrated response function

$$
\chi\left(t, t_{w}\right)=T \int_{t_{w}}^{t} R(t, s) d s .
$$

With respect to the usual definition, the temperature $T$ has been added in the above equation in order to simplify the notation in the following formulae and to have a well defined $\chi\left(t, t_{w}\right)$ in the $T \rightarrow 0$ limit. In the large time limit, Eq.(3) can be rewritten as

$$
\chi(C)=\int_{C}^{1} X(q) d q .
$$

So the FDR can be simply written as $X(C)=-\partial_{C} \chi(C)$.

The aim of this Letter is to propose and to show the efficacy of a very precise method for measuring the integrated response $\chi(C)$ and the FDR $X(C)$ in spin models.

Up to now the best protocol for measuring $\chi(C)$ in spin systems has been the following [3, 7]:

- initialize the system in a random configuration;

- quench the system at a temperature $T<T_{c}$ and evolve it for $t_{w}$ Monte Carlo sweeps (MCS);

- switch on a random magnetic field of very small intensity $h$ and continue evolving the system while measuring $C_{h}\left(t, t_{w}\right)$ and $\chi_{h}\left(t, t_{w}\right)$.

The parametric plot of $\chi_{h}\left(t, t_{w}\right)$ versus $C_{h}\left(t, t_{w}\right)$ converges to the function $\chi(C)$ in the limit $t_{w} \rightarrow \infty$ and $h \rightarrow 0$. Even when extrapolations can be safely done, they always require a large numerical effort: for example, in order to correctly take the $h \rightarrow 0$ limit, the whole simulation must be repeated for many $h$ values in the linear response regime. Moreover in frustrated systems like spin glasses the response may have strong non-linearities even for very small probing fields and it is usually very hard to predict a priori which is the linear response regime. Furthermore in out of equilibrium simulations the size of the linear response regime may change with the age 
of the system: A fair conjecture is that it decreases for larger times. If this would be true, extrapolations to the interesting limit would become still more difficult.

For all these reasons we consider of primary importance the development of a method which allows one to calculate the linear response in a spin system without applying any probing field. After having taken analytically the $h \rightarrow 0$ limit, one is left only with the $t_{w} \rightarrow \infty$ limit. This limit will be somehow unavoidable as long as the only way for aging a glassy system will be to simulate it for a long time [13.

Inspired by a recent work by Chatelain [9], we write down an analytical expressions giving the integrated response $\chi\left(t, t_{w}\right)$ in a simulation with no probing field 14].

Let us specialize on systems with $N$ Ising spins and Hamiltonian $\mathcal{H}_{0}$ (generalization to Potts variables is straightforward [8]). The Hamiltonian $\mathcal{H}_{0}$ may contain some quenched disorder, but we do not need to specify it, since our calculations hold for a generic $\mathcal{H}_{0}$, either disorder or not disordered. In the former case the final result can be eventually averaged over the quenched disorder distribution, but following formulae are valid for any given disorder realization.

When the probing field is switch on the Hamiltonian becomes $\mathcal{H}=\mathcal{H}_{0}-\sum_{i=1}^{N} h_{i} \sigma_{i}$, where $h_{i}$ are i.i.d. random variables with $\overline{h_{i}}=0$ and $\overline{h_{i} h_{j}}=h^{2} \delta_{i, j}$. For simplicity we define $h_{i}=h \varepsilon_{i}$ with $\overline{\varepsilon_{i}}=0$ and $\overline{\varepsilon_{i} \varepsilon_{j}}=\delta_{i, j}$.

The FDR for the observable $A(t)=\sum_{i} \varepsilon_{i} \sigma_{i}(t)$ is given in terms of the correlation and response functions

$$
\begin{aligned}
N C(t, s) & =\overline{\langle A(t) A(s)\rangle}=\sum_{i}\left\langle\sigma_{i}(t) \sigma_{i}(s)\right\rangle, \\
N R(t, s) & =\overline{\frac{\partial\langle A(t)\rangle}{\partial h(s)}}=\sum_{i} \frac{\varepsilon_{i} \sum_{j} \frac{\partial\left\langle\sigma_{i}(t)\right\rangle}{\partial h_{j}(s)} \frac{\partial h_{j}}{\partial h}}{\partial h}= \\
& =\sum_{i, j} \overline{\varepsilon_{i} \varepsilon_{j}} \frac{\partial\left\langle\sigma_{i}(t)\right\rangle}{\partial h_{j}(s)}=\sum_{i} \frac{\partial\left\langle\sigma_{i}(t)\right\rangle}{\partial h_{i}(s)},
\end{aligned}
$$

where $\langle\cdot\rangle$ represents the average over thermal histories.

We use a discrete-time dynamics as the one taking place in a Monte Carlo simulation. The time $t$ counts the number of single spin updates and not the number of
Monte Carlo sweeps (which is then $t / N$ ). The function $I(t)$ gives the index of the spin to be updated at time $t$, and so it depends on the updating rule (e.g. random or sequential). At the $t$-th time step the spin $\sigma_{i}$ with $i=I(t)$ is updated according to heat-bath probabilities

$$
\operatorname{prob}\left(\sigma_{i}=\sigma\right)=\frac{\exp \left[\beta \sigma\left(h_{i}^{\mathrm{W}}+h_{i}\right)\right]}{2 \cosh \left[\beta\left(h_{i}^{\mathrm{W}}+h_{i}\right)\right]},
$$

where $\beta$ is the inverse temperature and the Weiss field $h_{i}^{\mathrm{W}}$ takes into account the effect of Hamiltonian $\mathcal{H}_{0}$ on the spin to be updated. For example, in the case of 2spin interacting Hamiltonians the Weiss field is given by $h_{i}^{\mathrm{W}}=\sum_{j \neq i} J_{i j} \sigma_{j}$.

Under this dynamics the expectation value of the $j$-th spin a time $t$ is given by

$$
\left\langle\sigma_{j}(t)\right\rangle=\operatorname{Tr}_{\vec{\sigma}\left(t^{\prime}\right)}\left[\sigma_{j}(t) \prod_{t^{\prime}=1}^{t} W_{I\left(t^{\prime}\right)}\left(\vec{\sigma}\left(t^{\prime}\right) \mid \vec{\sigma}\left(t^{\prime}-1\right)\right)\right]
$$

where $\vec{\sigma}$ is a short-hand notation for the $N$ spins configuration, the trace is over all the trajectories $\vec{\sigma}\left(t^{\prime}\right)$ with $1 \leq t^{\prime} \leq t$, and the transition probability is given by

$$
W_{i}(\vec{\sigma} \mid \vec{\tau})=\frac{\exp \left[\beta \sigma_{i}\left(h_{i}^{\mathrm{W}}+h_{i}\right)\right]}{2 \cosh \left[\beta\left(h_{i}^{\mathrm{W}}+h_{i}\right)\right]} \prod_{j \neq i} \delta_{\sigma_{j}, \tau_{j}} .
$$

Note that $h_{i}^{\mathrm{W}}(\vec{\sigma})=h_{i}^{\mathrm{W}}(\vec{\tau})$ since it does not depend on the spin in $i$. The transition probability $W_{i}$ only depends on the perturbing field on site $i$, such that

$$
\left.\frac{\partial W_{i}(\vec{\sigma} \mid \vec{\tau})}{\partial h_{j}}\right|_{h=0}=\delta_{i, j} W_{i}(\vec{\sigma} \mid \vec{\tau}) \beta\left(\sigma_{i}-\sigma_{i}^{\mathrm{W}}\right),
$$

where we have defined $\sigma_{i}^{\mathrm{W}} \equiv \tanh \left(\beta h_{i}^{\mathrm{W}}\right)$.

Now we suppose that an infinitesimal probing field $h_{k}$ on site $k$ is switch on at time $t_{w}: h_{k}(t)=h \theta\left(t-t_{w}\right)$. This means that the transition probability $W_{k}$ (and only this one) will depend on the perturbing field for all times larger than $t_{w}$. Differentiation of Eq. (8) with respect to this field yields the integrated response

$$
\begin{aligned}
\chi_{j k}\left(t, t_{w}\right) & =\left.T \frac{\partial\left\langle\sigma_{j}(t)\right\rangle}{\partial h}\right|_{h=0}=\operatorname{Tr}_{\vec{\sigma}\left(t^{\prime}\right)}\left[\sigma_{j}(t) \prod_{t^{\prime}=1}^{t} W_{I\left(t^{\prime}\right)}\left(\vec{\sigma}\left(t^{\prime}\right) \mid \vec{\sigma}\left(t^{\prime}-1\right)\right) \sum_{s=t_{w}+1}^{t} \delta_{I(s), k}\left(\sigma_{k}(s)-\sigma_{k}^{\mathrm{W}}(s)\right)\right]= \\
& =\left\langle\sigma_{j}(t) \Delta \sigma_{k}\left(t, t_{w}\right)\right\rangle \quad \text { with } \quad \Delta \sigma_{k}\left(t, t_{w}\right)=\sum_{s=t_{w}+1}^{t} \delta_{I(s), k}\left(\sigma_{k}(s)-\sigma_{k}^{\mathrm{W}}(s)\right) .
\end{aligned}
$$

The correlation in Eq. (11) is what one has to measure in a numerical simulation with no perturbing field in order to get the integrated linear response.

Few comments are in order. The time-integrated quan- 


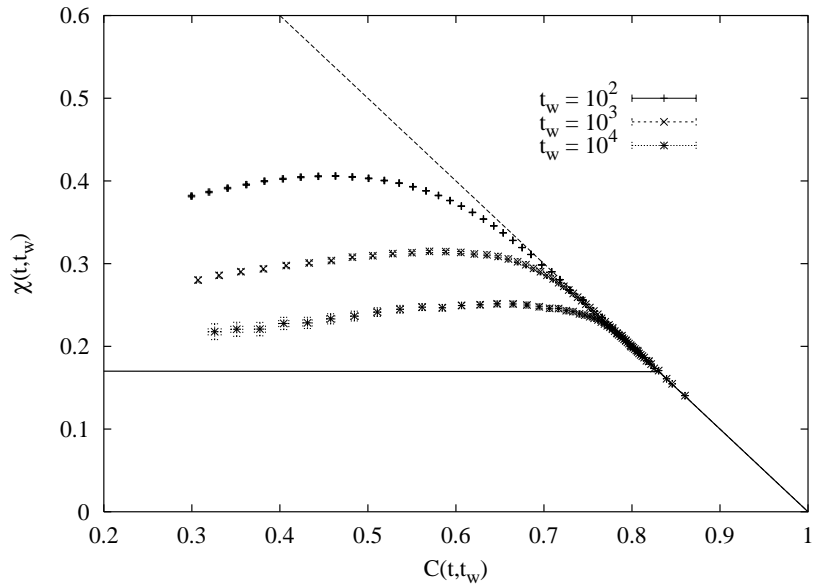

FIG. 1: FD plot for the 2D Ising ferromagnet at $T=2$. The horizontal line is the analytic long time limit.

tity $\Delta \sigma_{k}$ only gets contributions when the spin $\sigma_{k}$ is updated, so most of the times it is unchanged. The contributions summed up in $\Delta \sigma_{k}$ are the differences among the actual value of the spin $\sigma_{k}$ and the expected one $\sigma_{k}^{\mathrm{W}}$. So $\Delta \sigma_{k}$ is a random variable with zero mean, $\left\langle\sigma_{k}\right\rangle=\sigma_{k}^{\mathrm{W}} \Rightarrow\left\langle\Delta \sigma_{k}\right\rangle=0$, and variance $\left\langle\Delta \sigma_{k}^{2}\right\rangle \propto\left(t-t_{w}\right)$.

In the $T=0$ limit Eq. (11) has a nice and simple physical interpretation. Since for $T=0$ we have that $\sigma_{k}-\sigma_{k}^{\mathrm{W}}=\sigma_{k} \delta_{h_{k}^{\mathrm{W}}, 0}$, then $\Delta \sigma_{k}$ takes a contribution only when the spin $\sigma_{k}$ has a zero Weiss field on it, i.e. it is free to respond to an infinitesimal field. If the Weiss field is different from zero the spin is completely frozen and it can not respond to an infinitesimal perturbing field. So the integrated response in Eq.(11) can be rewritten as a simple sum of correlation functions $\chi_{j k}\left(t, t_{w}\right)=\sum_{s}^{\prime}\left\langle\sigma_{j}(t) \sigma_{k}(s)\right\rangle$, where the primed sum is over all the times larger then $t_{w}$ when $\sigma_{k}$ is updated under a zero Weiss field, i.e. being a free spin.

We now present numerical results for 3 spin models which are believed to belong to 3 different classes: ferromagnetic model in 2D (coarsening system), diluted longrange 3 -spin model with fixed connectivity 4 (discontinuous spin glass) and Edwards-Anderson model in 3D (continuous spin glass). For each model we have checked that the $\chi_{h}\left(C_{h}\right)$ curve measured with the perturbing field converges for $h \rightarrow 0$ to the one measured with the new method. Hereafter times will be counted in MCS.

The first model is the ferromagnetic Ising model on the 2-dimensional square lattice. We have simulated at $T=2 \simeq 0.88 T_{c}$ systems of sizes $1000^{2}$ and $7000^{2}$ in order to check the absence of any finite-size effect (the data we show are from the $1000^{2}$ samples). For each waiting time, $t_{w}=10^{2}, 10^{3}, 10^{4}$, averages have been taken over 100 different thermal histories, and the corresponding $\chi(C)$ curves are shown in Fig. 1] The horizontal line is the analytical prediction for the large times limit, $\chi=1-$ $m_{\mathrm{eq}}^{2}=0.17$. Numerical curves are clearly compatible

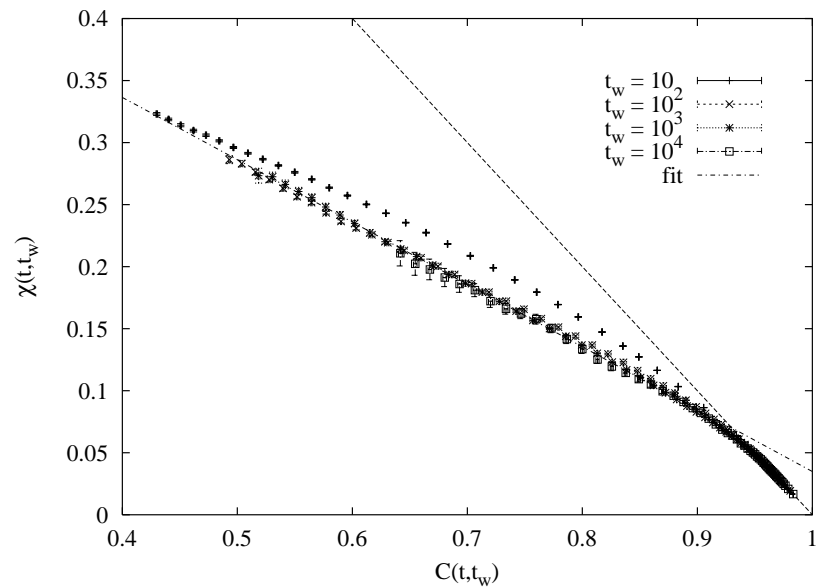

FIG. 2: FD plot for the long-range 3-spin model with fixed connectivity 4 at $T=0.5$. The line $0.53745-0.50256 C$ is the best linear fit to $t_{w}=10^{3}$ data with $C<0.9$.

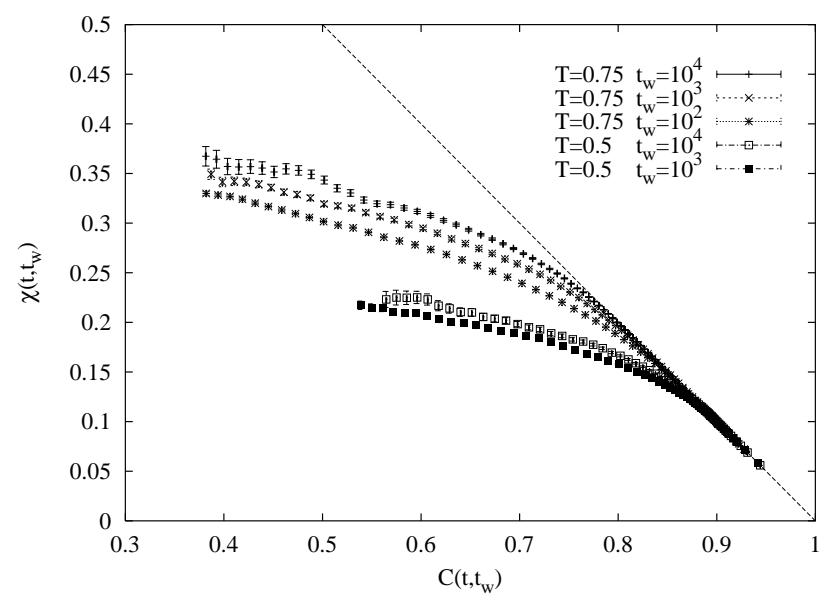

FIG. 3: FD plot for the 3D Edwards-Anderson model.

with the analytical asymptote in the large times limit.

The second model we studied is the 3 -spin model defined on a random hypergraph with fixed connectivity 4 . This model has been solved analytically with a one-step replica symmetry breaking Ansatz in Ref. 11]. The dynamical critical temperature is $T_{d}=0.755 \pm 0.01$. We have run simulations for a size $N=999999$ at temperature $T=0.5 \simeq 0.66 T_{d}$ and the resulting $\chi(C)$ curve is shown in Fig. 2 The number of samples used is 10 for $t_{w}=10,10^{2}, 50$ for $t_{w}=10^{3}$ and 20 for $t_{w}=10^{4}$. The straight line in Fig. 2] is a linear fit to $t_{w}=10^{3}$ data in the region $C\left(t, t_{w}\right)<0.9$, which perfectly interpolates the data $\left(\chi^{2} /\right.$ d.o.f. $\left.=0.82\right)$. It gives a Parisi breaking parameter on threshold states equal to $m_{\mathrm{th}}(T=0.5)=0.5 \pm 0.02$. The error is an estimate of systematic effects, mainly given by the slight increase of $m$ with $t_{w}$. Comparison of this value for $m_{\mathrm{th}}$ with corresponding static predictions will be done in Ref. [11].

The third model we studied is the 3 -dimensional 


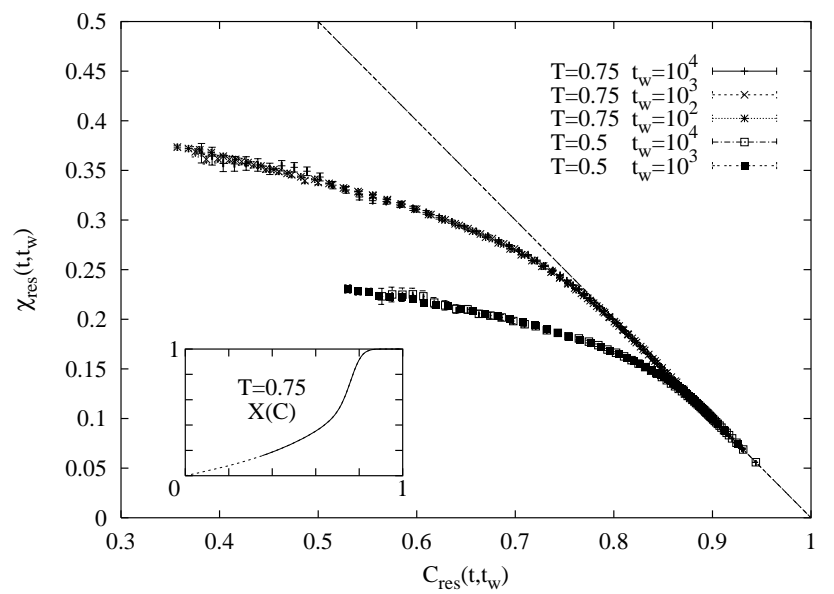

FIG. 4: Same as Fig. 3 with rescaled variables. Inset: FDR for $T=0.75$ obtained from the derivative of the rescaled data.

Edwards-Anderson model with $J= \pm 1$ couplings, which undergoes a phase transition to a spin glass phase at $T_{c}=1.14 \pm 0.01$ 10. We have simulated samples of size $L=20$ at temperatures $T=0.75 \simeq 0.66 T_{c}$ and $T=0.5 \simeq 0.44 T_{c}$, for three different waiting times $t_{w}=10^{2}, 10^{3}, 10^{4}$. The results are shown in Fig. [3

For a given temperature the $\chi(C)$ curves look very similar in shape, the main difference being the $t_{w}$-dependent Edwards-Anderson order parameter $q_{\mathrm{EA}}\left(t_{w}\right)$, here defined as the point where the $\chi(C)$ curve leaves the FDT line $1-C$. In order to exploit all the data we tried to collapse the curves before fitting. The collapse can be achieved either by shifting the curves such that the $q_{\mathrm{EA}}\left(t_{w}\right)$ coincide, either by the following rescaling: $C_{\mathrm{res}}\left(t, t_{w}\right)=\lambda C\left(t, t_{w}\right) / q_{\mathrm{EA}}\left(t_{w}\right), \chi_{\mathrm{res}}\left(t, t_{w}\right)=1-\lambda(1-$ $\left.\chi\left(t, t_{w}\right)\right) / q_{\mathrm{EA}}\left(t_{w}\right)$, with an arbitrary $\lambda$. Both scaling are

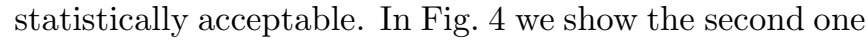
which is slightly better, with $\lambda=q_{\mathrm{EA}}\left(10^{4}\right)$.

If the measured data are already in the asymptotic regime, i.e. the scaling is valid for larger times, and since $\lim _{t_{w} \rightarrow \infty} q_{\mathrm{EA}}\left(t_{w}\right)=q_{\mathrm{EA}}>0$, we can conclude that the FDR is non trivial in the 3-dimensional EdwardsAnderson model, with an $X(C)$ like the one depicted in the inset of Fig. 4 for $T=0.75$.

The Edwards-Anderson model is the one which took the great part of the simulation time. Indeed, in order to have reasonable error bars, we ran at each of the two temperatures $10^{4}$ samples for $t_{w} \leq 10^{3}$ and almost $3 \cdot 10^{4}$ samples for $t_{w}=10^{4}$. Solely the $t_{w}=10^{4}$ runs took the equivalent of more than 1 year of CPU-time on a latest generation $2.0 \mathrm{GHz}$ computer. This is a consequence of the fact that errors on the linear response $\chi\left(t, t_{w}\right)$ increases like $\sqrt{t-t_{w}}$ and so the number of samples for keeping the error on $\chi(C)$ constant increases more or less linearly with the waiting time $t_{w}$. We believe this is the main drawback of the present new method for measuring the linear response: Although it is very successful for small times, it becomes very noisy at larger times and so it requires a huge statistics.

From this observation one could conclude that the usual old method of measuring the response with a small perturbing field would eventually remain the only valid one, but this is not true. Very probably the linear regime in the perturbing field $h$ decreases with the age of the system. In order to remain in the linear response regime one should decreases the intensity of the perturbing field during the simulation, thus increasing the error on the $\chi$ for late times.

Let us conclude with two remarks. First remark: Having understood that the integrated response can be written as a correlation function, it should be clear that all the functions $C\left(t, t_{w}\right)$ and $\chi\left(t, t_{w}\right)$ can be calculated in the same simulation for any value of $t$ and $t_{w}$. Moreover, being correlation functions self-averaging quantities, it should be possible in principle to calculate them in a single simulation of a sufficiently large sample. Second remark: The method presented here can be also used for any other Monte Carlo simulation (e.g. glass-former particle systems). The only condition for using present analytical expressions is the discreteness of time.

Acknowledgments Numerical simulations have been run on our Linux cluster IDRA. I thank ICTP for kind hospitality during the completion of this manuscript. Work supported in part by the European Community's Human Potential Programme under contract HPRN-CT2002-00307, Dyglagemem.

[1] For review see J.-P. Bouchaud et al., in Spin Glasses and Random Fields, A.P. Young ed. (World Scientific, 1997). L.F. Cugliandolo, preprint cond-mat/0210312.

[2] L.F. Cugliandolo and J. Kurchan, Phys. Rev. Lett. 71, 173 (1993); J. Phys. A 27, 5749 (1994).

[3] E. Marinari, G. Parisi, F. Ricci-Tersenghi, and J.J. RuizLorenzo, J. Phys. A 31, 2611 (1998).

[4] A. Crisanti and F. Ritort, J. Phys. A 36, R181 (2003).

[5] S. Franz, M. Mézard, G. Parisi, and L. Peliti, Phys. Rev. Lett. 81, 1758 (1998); J. Stat. Phys. 97, 459 (1999).

[6] L.F. Cugliandolo, J. Kurchan, and L. Peliti, Phys. Rev. E 55, 3898 (1997).

[7] A. Barrat, Phys. Rev. E 57, 3629 (1998).

[8] F. Krzakala and F. Ricci-Tersenghi, in preparation.

[9] C. Chatelain, preprint cond-mat/0303545.

[10] H.G.Ballesteros et al., Phys. Rev. B 62, 14237 (2000).

[11] A. Montanari and F. Ricci-Tersenghi, in preparation.

[12] We always consider correlation functions connected, i.e. $\lim _{t \rightarrow \infty} C(t, s)=0 \quad \forall s<\infty$, and normalized, $C(t, t)=1$.

[13] Accelerated dynamics can be used only in some particularly simple cases

[14] In the first version of Ref. 9], the only available at the time this work was finished, only the punctual response function $R(t, s)$ was studied and the calculations had some errors, making the final result unreliable. 